\title{
O papel dos eventos de vida em mães de crianças desnutridas: o outro lado da desnutrição
}

\author{
The role of life events in mothers of malnourished children: \\ the other side of malnutrition
}

Carmen Nudelmann ${ }^{1}$

Ricardo Halpern ${ }^{1}$

${ }^{1}$ Universidade Luterana do Brasil. Rua Miguel Tostes 101, São Luís. 92420-280 Canoas RS.

crmnudelmann@gmail.com
Abstract The objective of this article is to describe the life story of mothers of malnourished children and their possible associations to sociodemographic and psychosocial aspects. It is a cross sectional study carried out in a public health basic unit in Porto Alegre (RS, Brazil), with a total of 82 mothers. It was used a structured questionnaire with socio demographic, reproductive variables and mothers' live events. A descriptive anal$y$ sis and chi-square were used to investigate possible associations among the selected variables. Mothers had low schooling, low income and were multiparous besides, suffered parental abuse, had negative experiences with alcoholic parents (67\%), were neglect in infancy (65\%), have rejected the undernourished baby during pregnancy (70\%). They related depression symptoms during pregnancy (70\%) and postpartum (60\%). The study showed a difference of proportion between mothers who suffered abuse in childhood and those who not suffered according to pregnancy rejection $(p<0,001)$, depression during the pregnancy and after delivery $(p<0,001)$. This study associated mother's life stories with live events that, beyond the social and economic disadvantage, could develop a weak interaction between mother-baby that contribute to malnutrition.

Key words Childhood malnutrition, Maternal and child bonding, Alcoholism, Maternal life events
Resumo O objetivo deste artigo é descrever a história de vida de mães de crianças desnutridas e as possíveis relações com fatores sociodemográficos e psicossociais. Trata-se de estudo transversal realizado em unidade básica de saúde em Porto Alegre (RS) com 82 mães de crianças desnutridas. Usouse um questionário estruturado com variáveis sociodemográficas referentes à gestação e relacionadas à história de vida das mães. Realizaram-se análise descritiva e o teste Qui-quadrado para verificar possíveis associações entre as variáveis selecionadas. As mães apresentaram baixa escolaridade, baixa renda familiar, são multíparas, sofreram maus-tratos, experiências negativas com familiar alcoolista (67\%) e privação afetiva infantil (65\%); rejeitaram a gravidez do filho desnutrido (70\%); faltou apoio do companheiro na gestação e pósparto; $70 \%$ relataram sintomas depressivos no período gestacional e em torno de 60\% destas apresentaram os mesmos sintomas no pós-parto. Verificou-se diferença na proporção de mães que sofreram maus-tratos na infância e rejeição da gravidez $(p<0,001)$, sintomas depressivos na gravidez e pós-parto $(p<0,001)$. O estudo identificou na história de vida dessas mães fatores que, além da situação de desvantagem social e financeira, apresentam elementos para a formação de fraco vínculo mãe-bebê, contribuindo para a desnutrição.

Palavras-chave Desnutrição infantil, Vínculo mãe-bebê, Alcoolismo, Eventos de vida maternos 


\section{Introdução}

A Organização Mundial da Saúde (OMS) estima que cerca de 150 milhões de crianças menores de cinco anos, em todo o mundo, têm baixo peso para sua idade, e 182 milhões têm baixa estatura, sendo a desnutrição a segunda causa de morte mais frequente em menores de cinco anos nos países em desenvolvimento. Além disso, mais de $50 \%$ das mortes de crianças menores de cinco anos que ocorrem nesses países é influenciada pela desnutrição ${ }^{1}$.

A desnutrição infantil, apesar da redução mundial de sua prevalência, continua a ser o problema mais importante de saúde pública principalmente nos países mais pobres, devido a sua magnitude e consequências desastrosas para o crescimento, o desenvolvimento e a sobrevivência das crianças. E mais que um problema de saúde pública, a desnutrição, por ser de natureza multifatorial, passou a ser vista como um grave problema social ${ }^{1}$.

Celia et $a l^{2}$, em um trabalho de intervenção com bebês desnutridos em Porto Alegre (RS), verificaram que, além da carência alimentar, existe um distúrbio no vínculo mãe-bebê que, possivelmente, estaria associado à depressão materna. Este estudo, após a aplicação de um instrumento de avaliação de sintomas depressivos utilizando o Inventário de Beck, mostrou que a totalidade das mães de bebês desnutridos sofria algum grau de depressão. Nudelmann e Celia ${ }^{3}$, em estudo com mães de bebês desnutridos, sugerem a possibilidade de que as vivências dessas mulheres possam ter contribuído para a formação do vínculo mãebebê inadequado, favorecendo a ideia de um componente mais amplo, além da simples ingesta alimentar, para a compreensão da desnutrição infantil. Também Monte ${ }^{1}$ coloca a importância do vínculo mãe-filho como fator a mais na gênese da desnutrição, dizendo ser a desnutrição resultado de doenças, da dieta e de cuidados inadequados da mãe para com a criança. Através dessa visão, a síndrome de desnutrição é um problema de origem multifatorial, sendo o distúrbio no vínculo mãe-bebê apontado como um dos fatores associados à desnutrição.

A partir do questionamento sobre por que motivo, entre crianças de igual nível socioeconômico, algumas desnutrem e outras não, e das frequentes falhas nos programas de recuperação nutricional, surge a questão que busca identificar, na história de vida das mães, possíveis eventos que possam auxiliar a caracterizar e oferecer subsídios a propostas de programas de inter- venção que contemplem não somente os aspectos nutricionais, mas também os psicossociais ligados à desnutrição. Este estudo tem o propósito de descrever as características da história de vida das mães de crianças desnutridas que fazem acompanhamento no programa de recuperação nutricional em uma unidade básica de saúde em Porto Alegre (RS) e verificar as possíveis associações desses eventos com variáveis socioambientais e psicossociais.

\section{Material e métodos}

A amostra foi de 82 mães, todas participantes de um programa de vigilância do estado nutricional de crianças e gestantes da Prefeitura de Porto Alegre (RS, Brasil). O programa desenvolve-se em unidades básicas de saúde e contempla crianças em risco nutricional entre seis e sessenta meses de idade.

Para a montagem do instrumento de pesquisa, foram escolhidas inicialmente cinco mães de crianças desnutridas, que já haviam tomado parte do programa de recuperação nutricional do Vida Centro Humanístico, órgão do governo estadual, em parceria com a Prefeitura de Porto Alegre. A entrevistadora entrou em contato com cinco mães em suas residências, após estas serem escolhidas aleatoriamente da lista fornecida pelo programa e que concordaram em participar do estudo. Não houve recusas entre as mães convidadas. As mães entrevistadas nessa fase não participaram da segunda fase do estudo. Nessa etapa, a pesquisadora apresentou às mães uma única questão aberta: "Relate-me sua história de vida, fale-me tudo que a senhora pense ser importante e significativo desde a sua infância."

As entrevistas foram transcritas, e as informações fornecidas pelas mães foram analisadas através de uma análise de conteúdo e relacionadas para a montagem de um questionário estruturado. Além das questões coincidentes, foram selecionados situações e eventos críticos relatados pelas mães, e juntamente com o referencial teórico sobre o assunto elaborou-se um questionário estruturado, composto de 45 questões fechadas, abordando aspectos sociodemográficos, escolaridade, renda familiar, abandono e maustratos infantis, privação afetiva e nutricional e falta de apoio familiar sofridos pelas mães, além da história de alcoolismo familiar, rejeição da gestação e do bebê e sintomas depressivos maternos, utilizando-se como base o Inventário de Beck $^{4}$. 
Um banco de dados foi criado no programa Epi Info $6.0^{5}$, e realizou-se dupla digitação de dados. Os dados foram analisados no pacote estatístico SPSS $10.0^{6}$. Foi realizada uma análise descritiva e, para verificar as possíveis associações entre as variáveis selecionadas, foi aplicado o teste Qui-quadrado de Pearson.

\section{Resultados}

A maioria das mães é natural de Porto Alegre $(68,3 \%)$. Quanto à renda familiar, 73,2\% vivem com um valor igual ou menor que um salário mínimo (US\$140) e a maioria (60\%) frequentou a escola por mais de cinco anos. Oitenta por cento delas têm até quatro filhos. Em relação ao desejo de engravidar do filho desnutrido, $76 \%$ não desejaram a gestação.

Quase que a totalidade das mães realizou o pré-natal. Em sua maioria, o parto foi hospitalar $(93 \%)$, sendo que $76 \%$ delas tiveram o filho de parto normal. Os recém-nascidos, em sua maioria $(75 \%)$, tiveram peso classificado como insuficiente ao nascer entre $2.500-2.999 \mathrm{~g}$.

Em relação à história de vida, 55\% das mães relataram ter tido uma infância infeliz, por conta da falta de carinho de mãe e de pai e da presença de maus-tratos. A maioria das mães (53\%) foi cuidada pela própria mãe na infância, e aquelas que não foram cuidadas pela mãe apresentam como motivo o trabalho da mãe $(28 \%)$ e a rejeição da mãe (20\%).

Quanto ao tratamento dos cuidadores, $40 \%$ das mães referiram que eram agredidas com palavras, 30\% sofreram agressão física e $25 \%$ relataram falta de paciência dos cuidadores, sendo que, em alguns casos, esses eventos ocorreram de forma cumulativa.

Muitas mães (67\%) relataram ter convivido na infância com familiar alcoolista, sendo que, nesses casos, havia brigas na família, além de agressão física e verbal.

Em relação aos sentimentos vivenciados por elas quando souberam da gravidez desse filho, metade delas disse ter ficado triste, pois não queriam mais filhos e não tinham condições econômicas para sustentá-lo. Um terço dessas mulheres pensou em abortar e um terço relatou que a gravidez foi rejeitada pelo companheiro.

Sobre sentimentos vivenciados durante a gravidez, $60 \%$ das mães relataram tristeza, falta de ânimo e vontade de chorar, sendo que, depois do parto, em torno de $33 \%$ delas continuaram a vivenciar os mesmos sentimentos.
Ainda em relação às suas vivências (Tabela 1), $65 \%$ das mães disseram ter sofrido privação afetiva na infância, $67 \%$ sofreram maus-tratos pela mãe ou pelo pai quando crianças, $73 \%$ delas sentiram-se tristes e achavam que estavam com sintomas depressivos no período gestacional, e em torno de $44 \%$ disseram terem tido os mesmos sintomas após o nascimento do filho. Quase $60 \%$ das mães revelaram não ter tido nenhum apoio do companheiro durante a gestação, sendo que mais de $90 \%$ delas concordou que, para cuidar bem do bebê, a gestante e a mãe precisam sentir-se apoiadas. Grande parte das mães pensa que os seus bebês choravam por falta de carinho e por falta de atenção.

Setenta e oito por cento das mães que relataram uma infância infeliz tiveram um cuidador que as agredia fisicamente $(\mathrm{p}<0,007)$. A infância infeliz também está ligada ao convívio com familiar alcoolista $(\mathrm{p}<0,02)$. Entre as 45 mães que disseram ter sido infelizes na infância, quase $85 \%$ delas também não receberam apoio da sua mãe na gestação do filho desnutrido $(\mathrm{p}<0,02)$.

Em relação à rejeição da gravidez, entre as mães que disseram que tinham um cuidador que as agredia fisicamente, quase $70 \%$ delas também rejeitaram a gravidez $(\mathrm{p}<0,06)$.

Entre as mães que rejeitaram a gravidez com pensamento de aborto, $54 \%$ delas não tiveram apoio familiar durante a gestação $(\mathrm{p}<0,05)$, e 33\% tiveram sentimentos de tristeza no pós-parto $(\mathrm{p}<0,001)$.

Das mães que revelaram tristeza ao saber da gravidez, $68 \%$ disseram ter tido falta de carinho na infância $(p<0,001)$, e $69 \%$ relataram falta de apoio familiar na gestação $(\mathrm{p}<0,006)$.

Entre as mães que tiveram carência afetiva na infância, $80 \%$ apresentaram estado de ânimo depressivo na gravidez $(\mathrm{p}<0,001)$, e $66 \%$ tiveram sentimentos depressivos no pós-parto $(\mathrm{p}<$ 0,001). Entre as mães que se sentiram infelizes

Tabela 1. Sentimentos vivenciados pelas mães dos bebês desnutridos. Porto Alegre (RS), 2002.

$\begin{array}{lll}\text { Não teve carinho na infância } & \mathbf{5 3} & \mathbf{( 6 4 , 6 \% )} \\ \text { Sofreu maus-tratos pela mãe ou pelo pai } & 55 & (67,1 \%) \\ \text { Sintomas depressivos na gravidez } & 60 & (73,2 \%) \\ \text { Sintomas depressivos no pós-parto } & 36 & (43,9 \%) \\ \text { Não teve apoio do companheiro } & 49 & (59,8 \%) \\ \text { Mãe precisa de apoio do pai para cuidar do bebê } & 77 & (93,9 \%) \\ \text { Gestante precisa de apoio do pai para cuidar do bebê } & 75 & (91,5 \%) \\ \text { Acredita que o bebê chorava por falta de atenção } & 49 & (59,8 \%) \\ \text { Acredita que o bebê chorava por falta de carinho } & 39 & (47,6 \%)\end{array}$


durante a gestação, 74\% não tiveram apoio do companheiro $(\mathrm{p}<0,000)$.

\section{Discussão}

A partir dos resultados obtidos com este estudo, quando levamos em consideração as condições socioeconômicas das mães, e estas apresentam baixa renda familiar, identifica-se uma concordância com vários autores que enfatizam a importante associação entre a situação de pobreza da família e o estado nutricional das crianças ${ }^{1,7,8}$. Também a baixa escolaridade encontrada entre essas mães e o grande número de filhos são dados semelhantes àqueles encontrados em outros estudos com mães de crianças desnutridas ${ }^{9,10}$. Esses achados coincidem com aquilo que Parker et al. ${ }^{11}$ chamam de risco duplo. As crianças que vivem em países em desenvolvimento experimentam um duplo desafio. Primeiramente, são mais suscetíveis às doenças infectocontagiosas e, além disso, sofrem as consequências de viverem em condições sociais adversas, vivenciando distúrbios familiares e violência no seu cotidiano ${ }^{12-16}$.

O indicador de rejeição à gravidez encontrado entre as mães do estudo foi bastante significativo, estando a gravidez indesejada ligada por muitos autores a fatores importantes para a formação e o desenvolvimento do vínculo mãe-fi1 ho $\mathrm{O}^{2,10,17}$. Eles referem estar a desnutrição associada a uma falta de sincronia entre a mãe e o bebê, além da falta de alimento. Em estudo realizado na Índia, comparando crianças desnutridas e não desnutridas, foi encontrado que a conduta das mães dos desnutridos se caracterizava por menor receptividade e maior dificuldade de trocas no contato com os filhos ${ }^{18}$.

Além do desejo de engravidar e da aceitação da gravidez, o apoio recebido pela mãe durante o período gestacional está ligado à formação do vínculo mãe-bebe $\hat{e}^{19}$. Entre as mães do estudo, os resultados mostraram que a maioria delas não recebeu apoio do companheiro e também faltou apoio da família, mostrando coerência com trabalhos de autores que dizem que a falta de aceitação do bebê pelo companheiro da mãe, bem como a falta do seu apoio tanto no período prénatal como no perinatal, constitui risco adicional para o desenvolvimento do vínculo mãe-filho, podendo gerar um fator a mais para o surgimento da desnutrição ${ }^{2,20} \mathrm{e}$ afetar também o desenvolvimento no primeiro ano de vida ${ }^{21}$.

Quando nos reportamos à história de vida infantil das mães, é importante mencionar, entre os resultados obtidos, o significativo número de mães que sofreu agressões físicas, verbais e intolerância por parte de um dos cuidadores. Além disto, verificamos o fato de muitas mães terem tido envolvimento com familiar alcoolista e terem relatado a lembrança de muitas desavenças e agressões entre os familiares. Essas mães, quando questionadas sobre como haviam sido suas infâncias, responderam em sua maioria terem tido uma infância infeliz, tendo como motivos para tal sentimento a falta de carinho dos pais e a presença de maus-tratos. Os achados são semelhantes àqueles descritos por Nóbrega ${ }^{10} \mathrm{e}$ Klaus et al. ${ }^{19}$, que referem estar o papel de cuidador centrado nas experiências passadas da mãe, especialmente aquelas vividas com os próprios pais. Os resultados encontrados sugerem que, além das importantes questões ambientais como o contexto social em que a díade mãe-bebê vive, a qualidade da relação mãe-filho e as experiências infantis dos cuidadores devem ser levadas em consideração como cofator na determinação da desnutrição. Entre as mães do estudo, as agressões físicas e verbais sofridas na sua infância estão de acordo com estudo anterior que refere os pais de crianças desnutridas terem sido frequentemente vítimas de privações e abuso ${ }^{22}$.

A história de gestação das mães do estudo mostra que mais da metade delas referiram tristeza ao saber da gravidez e não queriam mais filhos. Grande parte delas pensou em abortar e um número significativo relatou rejeição à gravidez por parte do companheiro. Elas consideram que, para cuidar bem do bebê, tanto a gestante como a mãe precisam ser apoiadas, indo ao encontro do que dizem alguns autores no sentido de que quando as mães recebem apoio do companheiro, têm autoestima mais elevada e apresentam menos sintomas depressivos ${ }^{23,24}$.

Quando se investigam sentimentos relacionados a sintomas depressivos entre essas mães, percebe-se que mais da metade delas apresenta vontade de chorar, tristeza e falta de ânimo, sendo que mais de $70 \%$ disseram ter estado tristes durante o período gestacional e quase $50 \%$ revelaram ter tido o mesmo sentimento após o parto. Autores como Galler et al. ${ }^{25}$, Nudelmann ${ }^{26}$ e Celia e Nudelmann ${ }^{27}$ sustentam a ideia de que sintomas depressivos e sentimentos de falta de esperança ocorrem mais frequentemente entre mães de crianças desnutridas quando comparadas com mães de crianças eutróficas. O estado de ânimo dessas mulheres estaria ligado à pobreza, falta de suporte social e familiar, altos níveis de estresse, bem como aumento de frustrações e maus-tratos infantis. 
Em relação aos motivos que provocam o choro do bebê, mais da metade das mães referiu que o seu bebê chorava porque faltava atenção da própria mãe, e quase metade das mães relacionou o choro do bebê à falta de carinho. Esses achados são descritos por Klaus et al. ${ }^{19}$, que fazem referência a bebês que, quando mantidos junto ao corpo do cuidador por menos tempo, metade costuma chorar mais do que aqueles bebês que recebem mais colo e atenção. Frank e Zeisel $^{22}$ indicam que na desnutrição existe um sistema familiar com funcionamento inadequado que, somado à situação de pobreza e a fatores estressantes, como discórdia entre os pais, alcoolismo e humor deprimido, circunstâncias essas encontradas entre as mães do estudo, levam a uma maior dificuldade para cuidar da criança, determinando um cuidado ao bebê deficiente em carinho e atenção.

Quanto à história de vida infantil dos cuidadores e sua relação com sentimentos vivenciados pelas mães, é importante salientar que a análise mostrou uma diferença estatisticamente significativa em relação à presença de maus-tratos na infância e à percepção de infância infeliz registrada pelas mães. Da mesma forma, houve uma diferença estatisticamente significativa quanto à presença e convívio com familiar alcoolista e ambiente agressivo e o sentimento das mães de terem tido uma infância infeliz. Esses achados são coerentes com os estudos de Bowlby ${ }^{28}$, quando o autor faz referência àquelas mães que tiveram experiências adversas na infância e que cresceram ansiosamente apegadas e tendem a repetir tais modelos com seus filhos. Nesses casos existe uma probabilidade maior entre as mulheres que sofreram negligências e ameaças de abandono na infância de também negligenciarem seus filhos.

A análise dos sentimentos vivenciados pelas mães na gestação relacionados ao comportamento do cuidador na infância revela magnitude da diferença entre as proporções entre cuidador agressivo, sob qualquer forma de agressão, e a rejeição da gravidez com pensamento de aborto. Também a tristeza da mãe ao saber da gravidez mostrou uma diferença estatisticamente significativa entre a proporção de mães que tiveram falta de carinho na infância e aquelas que não o tiveram.

O desejo de abortar, a tristeza ao saber da gravidez e os sentimentos de tristeza pós-parto marcaram uma associação com falta de apoio familiar. Celia e Nudelmann ${ }^{27}$ apontam como um dos fatores favorecedores de sintomas depressivos entre mães de bebês desnutridos a falta de apoio familiar. Junta-se ao exposto a contribui- ção de Silva et al. ${ }^{20}$, que apresentam como fator relevante de indução a esses sintomas a falta de apoio afetivo do companheiro da mãe.

O fato de as mães terem tido ou não carinho na infância e sentimentos de ânimo depressivo na gravidez do filho desnutrido também mostrou resultado estatisticamente significativo e coerente com Bowlby ${ }^{28}$, que fala da existência de uma ruptura entre a pessoa apegada e a figura de apego, portanto entre a mãe e a mãe da mãe, gerando dor e depressão. Essa deficiência de carinho na infância leva à maior possibilidade de a mulher, quando em período gestacional e pósnatal, desenvolver sintomas depressivos.

Sobre a presença de sentimentos relacionados com sintomas depressivos no período gestacional e a proporção de mães que rejeitaram a gravidez, a análise foi estatisticamente significativa, sugerindo a ideia de rejeição ao bebê desnutrido, ligada à depressão materna e favorecendo a formação de um fraco vínculo mãe-filho ${ }^{2,10}$. Outro resultado que aparece significativo é aquele que relaciona a falta de apoio do companheiro e a presença de sintomas depressivos na gestação do filho desnutrido. Os achados são semelhantes àqueles encontrados em estudo sobre depressão pré e pós-natal com mulheres brasileiras de baixa renda, mostrando que as mulheres grávidas que tiveram depressão receberam menos apoio do companheiro em comparação com aquelas que não sofreram depressão ${ }^{20}$.

A desnutrição é um problema mundial de saúde pública de causa multifatorial, envolvendo pobreza, problemas de dieta, doenças, serviços de saúde deficientes e cuidados inadequados da mãe ${ }^{1}$, bem como risco maior de atraso no desenvolvimento neuropsicomotor das crianças atingidas pela síndrome $e^{8,21}$. Nessa gama de fatores que envolvem a desnutrição, não podem ser deixados de lado aqueles de ordem psicossocial, entre eles o vínculo mãe-filho e as vivências infantis dos cuidadores.

O estudo, por ser eminentemente descritivo, apresenta as limitações inerentes ao delineamento. Apesar disso, foi possível realizar análises exploratórias, com resultados consistentes a ponto de permitir relacionar as situações adversas vivenciadas pelas mães na sua infância e período gestacional com a possibilidade de esses fatores contribuírem nas questões referentes à nutrição de seus filhos. Esses achados podem ser decorrentes de causalidade reversa, limitação comum de estudos transversais. Certamente, o tamanho amostral deve ter contribuído para que alguns achados mostrassem uma significância marginal, 
ou não mostrassem associação onde na realidade ela poderia existir caracterizando um erro tipo II. Mas o fato de que algumas associações apareceram significativas reforça a ideia de que são importantes na construção do modelo teórico multifatorial da desnutrição infantil. Serão necessários estudos com um número maior de sujeitos que permitam que as diversas variáveis possam ser controladas através de uma análise multivariada e possam oferecer a medida exata de cada variável na determinação da desnutrição infantil.

As crianças de países em desenvolvimento sofrem o que foi descrito por Bobadilla e Pos$\operatorname{sas}^{29}$ como "dupla carga de doenças" com a exposição aos riscos tradicionais e também aos riscos modernos. Desde o início da década de 70, pesquisas demonstraram que o processo causador da desnutrição infantil não se fixa na quantidade de calorias ingeridas pela criança, mas é parte de um processo que inclui transformações sociais amplas e um atendimento global à saúde da criança ${ }^{30}$. A mudança do conceito de desnutrição levou entre outras coisas à promoção de uma abordagem mais ampla do problema, le- vando em conta variáveis socioeconômicas psicossociais e demográficas envolvidas. Esta visão contextual de um dos problemas críticos da saúde infantil levou a uma análise mais abrangente em relação à promoção de saúde da criança. Além das situações do risco biológico, existe a possibilidade de as crianças vivenciarem os estresses relativos às condições desfavoráveis de vida, propiciados, na sua maioria, pelo aumento da urbanização, violência, mudanças na estrutura familiar e, em algumas áreas específicas, pela diminuição do suprimento alimentar e pelo fato de que os avanços nos cuidados demoram mais a chegar para as crianças desfavorecidas ${ }^{31,32}$.

Nesse contexto e tendo em mente os efeitos deletérios que a desnutrição pode causar na criança, a identificação de situações ligadas à história de vida das mães pode auxiliar de forma decisiva aquelas crianças com risco nutricional. A partir disso, supõe-se que programas de intervenção nutricional poderão obter resultados mais satisfatórios quando valorizada a história de vida das mães como fatores coadjuvantes no processo de determinação da desnutrição infantil.

\section{Colaboradores}

C Nudelmann foi responsável pela coleta dos dados, pelo controle de qualidade, revisão bibliográfica e primeira versão do artigo; R Halpern foi responsável pela análise estatística, primeira versão e revisão do artigo. 


\section{Referências}

1. Monte CMG. Desnutrição: um desafio secular à nutrição infantil. J Pediatria 2000; 76(Supl.3):285296.

2. Celia S, Alves M, Behs B, Nudelmann C, Saraiva J. Relatório da Plenária do V Congresso WAIPAD. Chicago, EUA; 1992.

3. Nudelmann C, Celia S. Relatório do Clinical Teaching: in the face of the gorgan: creative home interventions with hard to reach families with infants home visits. Sixth World Congress of the World Association for Infant Mental Health. Tampere, Finlândia; 1996.

4. Cunha JA. Generalidades sobre a versão brasileira do BDI, BAI, BHS e BSI. 1' Congresso Ibero-Americano de Avaliação Psicológica. Anais do $7^{\circ}$ Encontro Nacional sobre Testes Psicológicos. Porto Alegre; 1997. p. 135-139.

5. Dean AG, Dean JA, Coulombier D. Epi Info, Version 6.04: a word processing, database, and statistics program for public health on IBM-compatible microcomputers. Atlanta: Centers for Disease Control and Prevention; 1996.

6. Norussis NJ. Statistical package for social sciences for Windows. Chicago: SPSS; 1993.

7. Halpern R. Poverty and early childhood parenting: toward a framework for intervention. American Journal of Orthopsychiatry 1990; 60(1):101-116.

8. Cravioto J, Milán RA, Villicaña R. Desnutrição e sistema nervoso central. In: Diament A, Cypel S. Neurologia infantil. 3a ed. São Paulo: Atheneu; 1998. p. 1084-1099.

9. Solymos GMB. A experiência vivida de mães de desnutridos: um estudo fenomenológico de fatores psicossociais de risco [dissertação]. São Paulo: Instituto de Psicologia da Universidade de São Paulo; 1995.

10. Nóbrega FJ. Evolução a longo prazo do desnutrido grave. Pediatria Moderna 1997; 33(7):143-166.

11. Parker S, Greer S, Zuckermann B. Double jeopardy: the impact of poverty on early child development. Pediatrics Clinics of North America 1988 35(6):1227-1240.

12. Costello EJ, Compton SN, Keeler G, Angold A. Relationships between poverty and psychopathology. JAMA 2003; 290(15):2023-2029.

13. Nair MK, Radhakrishnan R. Early childhood development in deprived urban settlements. Indian Pediatrics 2004; 41:227-237.

14. Halpern R, Karam S, Mulinari E, Horta B, Riggo A Tomasi E. Desenvolvimento e comportamento in fantil: uma visão dos pediatras. Revista Brasileira de Educação Médica 1997; 21(1):33-38.

15. Escalona SK. Babies at double hazard: early development of infants at biologic and social risk. Pediatrics 1982; 70(5):670-675.

16. Andraca I, Pino P, La Parra A, Rivera F, Castillo M. Factores de riesgo para desarrollo psicomotor en lactentes nacidos en optimas condiciones biológicas. Rev Saude Publica 1988; 32(2):138-147.

17. Ferreira MCR. Malnutrition and mother infant asynchrony: slow mental development. J Behavioral Development 1978; 1:207-209.
18. Graves PL. Nutrition, infant behavior, and maternal characteristics: a pilot study in West Bengali, India. Am J Clin Nutr 1976; 29:305.

19. Klaus MH, Kennel JH, Klaus PH. Vínculo. Porto Alegre: Artmed; 2000.

20. Silva VA, Moraes ARS, Carvalho MS, Martins ML, Teixeira NA. Prenatal and postnatal depression among low income Brazilian women. Brazilian Journal Medical Biology 1998; 31(6):799-804

21. Halpern R, Giugliani ER, Victora CG, Barros FC, Horta BL. Risk factors for suspicion of developmental delays at 12 months of age. J Pediatr (Rio J) 2000; 76:421-428.

22. Frank DA, Zeisel SH. Failure to thrive. The Pediatric Clinics of North America 1988; 35(6):1187-1206.

23. Murata A, Nadaoka T, Morioka Y, Oiji A, Saito H Prevalence and background factors of maternity blues. Gynecology Obstetric Investigation 1998; 46(2):99-104

24. Navaie-Waliser M, Martin SL, Tessaro I, Campbell MK, Cross AW. Social support and psychological functioning among high-risk mothers: the impact of the baby love maternal outreach worker program. Public Health Nurse 2000; 17(4):280-291.

25. Galler JR, Salt P, Ramsey FC. The influence of early malnutrition on subsequent behavioral: the effects on maternal depressive symptoms. J Dev Behav Pediatrics $1988 ; 9(1): 1-4$

26. Nudelmann C. Reações do bebê à depressão materna. In: Pitrez JLB, Picon PX. Pediatria consulta rápida. Porto Alegre: Artes Médicas; 1999. p. 130-131.

27. Celia S, Nudelmann C. Evaluation of an intervention program in the diad depressive mother-undernourished baby and its follow-up. Medical News, Special Psychiatry 2001; 11(2):63-67.

28. Bowlby J. Uma base segura. Porto Alegre: Artes Médicas; 1989

29. Bobadilla JL, Possas CA. Health policy issues in three Latin American countries: implications of the epidemiological transition. In: Gribble JN, Preston SH, editors. The epidemiological transition: policy and planning implications for developing countries. Washington: National Academy Press; 1993. p. 145-169.

30. Giugliani ER, Seffrin CF, Goldan M, Horn JF, Ebrahim GJ. The malnourished children of the urban squatter families: a study in Porto Alegre, Brazil. J Trop Pediatr 1987; 33(4):194-198.

31. Engle PL, Castle S, Menon P. Child development: vulnerability and resilience. Soc Sci Med 1996; 43(5):621-635.

32. Victora CG, Vaughan JP, Barros FC, Silva AC, Tomasi E. Explaining trends in inequities: evidence from Brazilian child health studies. Lancet 2000; 356:1093-1098.

Artigo apresentado em 19/09/2008

Aprovado em 17/04/2009

Versão final apresentada em 17/05/2009 